\title{
Síndrome de Cushing exógeno de difícil diagnóstico
}

\section{Exogenous Cushing's syndrome with a difficult diagnosis}

\author{
Luis Felipe Fierro-Maya, Angélica Maria González-Clavijo, Miguel Ángel Álvarez-Lozano, \\ Gonzalo Alejandro Moreno-Soler, Sebastián Rodríguez-Paz, Daniela Tarquino- Rincón \\ - Bogotá, D.C. (Colombia)
}

DOI: https://doi.org/10.36104/amc.2020.1500

\section{Resumen}

Introducción: el síndrome de Cushing (SC) es un grupo de signos y síntomas causados por la exposición crónica al exceso de glucocorticoides. El uso de fármacos con glucocorticoides es la causa más frecuente, pero algunos productos vendidos como suplementos nutricionales o medicamentos naturistas para el control de los dolores articulares pueden contenerlos de forma oculta, lo cual dificulta el enfoque diagnóstico

Presentación del caso: paciente de 40 años con signos clínico típicos de síndrome de Cushing con resultados discordantes en las pruebas diagnósticas para identificar su origen. Finalmente se logra establecer que el paciente consumía de forma crónica un producto "naturista" conocido como artrin ${ }^{\circledR}$, el cual fue analizado en el laboratorio de la institución con resultados positivos para cortisol.

Conclusión: la exposición crónica a glucocorticoides exógenos, ya sea subrepticia, desconocida o prescrita, causa SC y debe distinguirse tempranamente de las formas endógenas para evitar la realización de pruebas diagnósticas y tratamientos inadecuados. (Acta Med Colomb 2020; 45. DOI: https://doi.org/10.36104/amc.2020.1500).

Palabras clave: síndrome de Cushing, glucocorticoides exógenos, suplementos herbales.

\section{Abstract}

Introduction: Cushing's syndrome (CS) is a group of signs and symptoms caused by chronic exposure to excessive glucocorticoids. The use of medications containing glucocorticoids is the most common cause, but they may be hidden in some products sold as nutritional supplements or naturopathic medications, which makes the diagnostic approach more difficult.

Case presentation: this was a 40-year-old patient with typical clinical signs of Cushing's syndrome and discordant results of diagnostic tests to identify its origin. It was ultimately determined that the patient had been chronically taking a "naturopathic" product known as artrin®, which was analyzed in the institution's lab and found to contain cortisol.

Conclusion: chronic exposure to exogenous glucocorticoids, whether surreptitious, unknown or prescribed, causes CS and should be promptly distinguished from endogenous forms to avoid inappropriate diagnostic tests and treatments. (Acta Med Colomb 2020; 45. DOI: https://doi. org/10.36104/amc.2020.1500).

Key words: Cushing's syndrome, exogenous glucocorticoids, herbal supplements.
Dr. Luis Felipe Fierro-Maya: Instituto Nacional de Cancerología, Unidad de Endocrinología Oncológica; Dra. Angélica María González-Clavijo: Instituto Nacional de Cancerología, Unidad de Endocrinología Oncológica. Universidad Nacional de Colombia, Facultad de Medicina, Departamento de Ciencias Fisiológicas, Unidad de Bioquímica; Dres. Miguel Ángel ÁlvarezLozano, Gonzalo Alejandro Moreno-Soler, Sebastián Rodríguez-Paz, Daniela TarquinoRincón: Universidad Nacional de Colombia, Facultad de Medicina, Departamento de Ciencias Fisiológicas, Unidad de Bioquímica. Bogotá, D.C. (Colombia).

Correspondencia: Dra. Angélica María González-Clavijo. Bogotá, D.C. (Colombia).

E-mail: angelik_md@yahoo.com

Recibido: 31/VII/2019 Aceptado: 13/04/2020

\section{Introducción}

El síndrome de Cushing (SC) es una enfermedad poco frecuente con una incidencia de dos a tres casos por millón de personas por año, pero con alta morbimortalidad (1). Es causado por la exposición prolongada e inapropiada al exceso de glucocorticoides. La presencia de signos físicos tales como las facies de luna llena, la obesidad troncular, estrías violáceas, hirsutismo y acné, así como las complicaciones metabólicas asociadas como la diabetes, hipertensión arterial y osteoporosis, deben hacer sospechar hipercortisolismo; sin embargo, el diagnóstico casi siempre constituye un reto clínico. 
Existen dos tipos de síndrome de Cushing: el endógeno y el exógeno. El síndrome de Cushing endógeno puede ser ACTH dependiente, que corresponde a 80-85\% de los casos, originado en la mayoría de los casos en un adenoma hipofisario secretor de ACTH o causado por la secreción ectópica de ACTH o de hormona liberadora de corticotropina (CRH) en ciertos tumores neuroendocrinos pulmonares o pancreáticos. El síndrome de Cushing ACTH independiente abarca el 15-20\% de los casos de hipercortisolismo endógeno y es consecuencia de adenomas o carcinomas adrenales (2-4).

El Cushing exógeno, que es la principal causa de hipercortisolismo, es consecuencia de la exposición a medicamentos con efecto glucocorticoide por tiempo prolongado. Generalmente su curso es transitorio y se resuelve con la suspensión de la exposición, sin embargo, cuando se consumen productos que de manera oculta contienen glucocorticoides, se puede convertir en una enfermedad crónica de difícil diagnóstico (5).

\section{Caso clínico}

Paciente de 40 años con antecedente de artritis psoriásica desde los 30 años de edad, quien desde un año antes de la consulta presentó cambios físicos con obesidad central, estrías violáceas abdominales y facies de luna llena, asociados a diabetes mellitus de difícil control glucémico e hipertensión arterial severa. Fue estudiado en su sitio de residencia habitual encontrando hipercortisolismo. El paciente negó en repetidas ocasiones el consumo de fármacos glucocorticoides.

Fue remitido al Instituto Nacional de Cancerología con diagnóstico de síndrome de Cushing ACTH dependiente, con imagen de hipófisis negativa para adenomas. En la valoración inicial se decidió repetir las pruebas bioquímicas (Tabla 1), debido a que sus resultados no fueron concordantes. Los resultados de laboratorio institucionales se muestran también en la Tabla 1, con hallazgos indicativos de síndrome de Cushing ACTH independiente. Se practicó una tomografía de suprarrenales que fue normal.

Posteriormente en una segunda visita se encontró un cortisol basal suprimido, lo que hizo sospechar la ingesta subrepticia de glucocorticoides. El paciente manifestó que tomaba de manera intermitente desde hace más de 12 meses un producto "naturista" comercializado como artrin ${ }^{\circledR}$, con tiempos de uso continuo por tres a cuatro meses y períodos sin uso por hasta dos semanas. El artrin ${ }^{\circledR}$ es un producto que se comercializa en Colombia sin registro sanitario INVIMA (6); sin embargo en la etiqueta no se mencionan glucocorticoides como parte de su composición, como se muestra en la Figura 1. Debido a la coincidencia temporal entre el consumo de $\operatorname{artrin}{ }^{\circledR}$ y el cuadro clínico y a la ausencia de información sobre su contenido en las fuentes oficiales (INVIMA), realizamos una prueba de determinación de cortisol en el contenido de tableta de artrin ${ }^{\circledR}$. Para ello se diluyó una tableta en $5 \mathrm{cc}$ de solución salina normal y se tomó una alícuota, la cual se procesó en el laboratorio clínico de nuestra institución empleando el ensayo de cortisol elecsys ${ }^{\circledR}$ de primera generación (Roche Diagnostics) con un resultado mayor a $1352 \mathrm{nmol} / \mathrm{L}$ (quimioluminiscencia, rango $<5-1352 \mathrm{nmol} / \mathrm{L}$ ).

Se indicó suspender el consumo del producto "naturista" y para evitar la insuficiencia adrenal por supresión del eje, el paciente fue suplido con prednisolona oral $5 \mathrm{mg} / \mathrm{día}$ con reducción gradual de la dosis hasta su retiro completo en seis semanas, con la mejoría progresiva de las características físicas de Cushing y corrección completa de la hiperglucemia aunque persistió con hipertensión arterial.

\section{Discusión}

El síndrome de Cushing (SC) es un grupo de signos y síntomas causados por la exposición crónica al exceso de glucocorticoides. En el abordaje diagnóstico de todo pa-

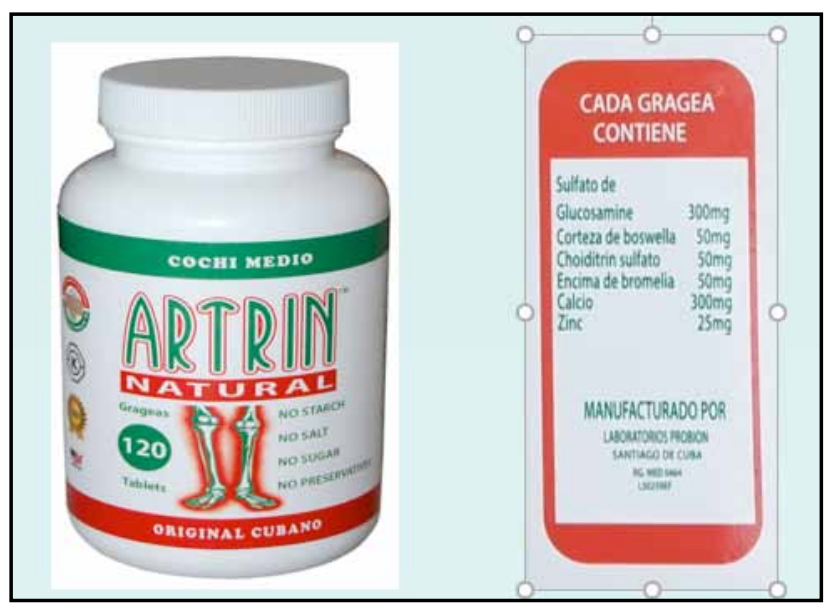

Figura 1. Presentación y etiqueta del producto artrin $®$.

Tabla 1. Pruebas de laboratorio clínico del paciente.

\begin{tabular}{|l|c|c|c|}
\hline \multicolumn{1}{|c|}{ Mes } & Cortisol A.M & ACTH A.M & Cortisol TSDDB \\
\hline Agosto 2008 (externas) & 9.0 & 82 & 3.9 \\
\hline Noviembre 2008 (INC) & 8.07 & Menor a 1 & 10.9 \\
\hline Marzo 2009 (INC) & 3.4 & SD & SD \\
\hline SD: Sin dato & TSDDB: Test supresión de cortisol con dexametasona dosis bajas. & SD \\
\hline
\end{tabular}


ciente con clínica de hipercortisolismo es imperativo que se descarte el consumo de medicamentos que contengan glucocorticoides. Estos fármacos se recetan comúnmente para uso por vía oral, parenteral, tópica, rectal o inhalada. Puede ocurrir que los pacientes que reciben inyecciones para el control del dolor no sean conscientes de que han recibido un potente glucocorticoide. Los glucocorticoides también pueden estar presentes en una variedad de medicamentos que son vendidos sin receta. Recientemente se ha reportado el uso de cremas blanqueadora de la piel y su asociación con síndrome de Cushing (7).

Existen productos comercializados sin receta que mencionan contener extractos de plantas con propiedades analgésicas y antiinflamatorias. Si bien los extractos de bromelia que contienen glucósidos flavonoides, pueden tener propiedades anti inflamatorias (8), al igual que los extractos de boswellia serrata (9), en el caso del producto artrin $\AA$ usado por el paciente inferimos que se trata de un producto fraudulento, pues en la etiqueta se observa una redacción incorrecta de los extractos de las plantas que dice contener y sobre todo porque el contenido de glucocorticoides quedó evidenciado por la detección de cortisol en la prueba de laboratorio clínico.

Cuando un paciente que usa este tipo de productos presenta características de síndrome de Cushing se aconseja la medición de los esteroides sintéticos en sangre u orina (10). No obstante, en nuestro medio no están disponibles las pruebas de laboratorio para determinar las concentraciones de tales esteroides sintéticos, por ello nos basamos en la posibilidad de los glucocorticoides sintéticos como la prednisolona, prednisona y metilprednisolona, de generar reactividad cruzada con la prueba de cortisol en plasma y orina por métodos de inmunoensayo como se muestra en la Tabla 2. Por el contrario la dexametasona, genera poca o ninguna reacción cruzada con el cortisol en los ensayos analíticos, lo que justifica su uso para la realización de la prueba diagnóstica de tamización de síndrome de Cushing conocida como test de supresión con dosis bajas de dexametasona (11).

Tabla 2. Reactividad cruzada en pruebas de inmunoensayo para cortisol.

\begin{tabular}{|c|c|c|}
\hline Esteroides sintéticos & \multicolumn{2}{|c|}{ Reactividad cruzada en la prueba de cortisol } \\
\hline & $\begin{array}{c}\text { Por quimioluminiscencia } \\
\text { (12) }\end{array}$ & $\begin{array}{c}\text { Por inmunoensayo } \\
\text { enzimático ELISA (13) }\end{array}$ \\
\hline Metil prednisolona & $26 \%$ & $249 \%$ \\
\hline Prednisolona & $109 \%$ & $148 \%$ \\
\hline Prednisona & $39 \%$ & $0.3 \%$ \\
\hline Dexametasona & $0.2 \%$ & NR \\
\hline NR: No reportado & \\
\hline
\end{tabular}

Con los resultados de la prueba sospechamos que artrin $\AA$ contiene alguno de los tres esteroides sintéticos mencionados; sin embargo, ante el INVIMA, artrin ${ }^{\circledR}$ figura como un producto cuya composición legítima es desconocida (6).

La discordancia entre las pruebas de cortisol sérico y ACTH observada en este paciente se explicó por la toma intermitente del producto artrin ${ }^{\circledR}$ y por el empleo de métodos analíticos distintos en las diferentes mediciones.

\section{Conclusión}

La exposición crónica a glucocorticoides exógenos debe descartarse tempranamente en todo paciente con sospecha de síndrome de Cushing, y deben tenerse en cuenta los "medicamentos naturistas", sobre todo aquellos con "propiedades antinflamatorias y analgésicas", dado que de manera oculta pueden contener esteroides sintéticos.

\section{Referencias}

1. Hagen C, Jorgensen J, Kosteljanetz M, Kristensen L, Laurberg P, Schmidt K Weeke J. 2001 Incidence and late prognosis of Cushing's syndrome: a populationbased study. J Clin Endocrinol Metab 86:117-123 [PubMed] [Google Scholar]

2. Pivonello R, De Martino MC, De Leo M, Lombardi G, Colao A. Cushing's síndrome Endocrinol Metab Clin North Am. 2008 Mar;37(1):135-49, ix. doi: 10.1016/j.ecl.2007.10.010.

3. Lacroix A, Feelders RA, Stratakis CA, Nieman LK. Cushing's síndrome. Lancet. 2015 Aug 29;386(9996):913-27. doi: 10.1016/S0140-6736(14)61375-1. Epub 2015 May 21.

4. G.Arnaldi, A.Angeli,A. B.Atkinson, X. Bertagna, F. Cavagnini, G.P.Chrousos, G. A. Fava, J. W. Findling, R. C. Gaillard, A. B. Grossman, B. Kola, A. Lacroix, T. Mancini, F. Mantero, J. Newell-Price, L. K. Nieman, N. Sonino, M. L. Vance, A. Giustina, M. Boscaro, Diagnosis and Complications of Cushing's Syndrome: A Consensus Statement, The Journal of Clinical Endocrinology \& Metabolism, Volume 88, Issue 12,1 December 2003, Pages 5593-5602, https:// doi.org/10.1210/jc.2003-030871

5. Wei L, MacDonald TM, Walker BR. 2004 Taking glucocorticoids by prescription is associated with subsequent cardiovascular disease. Ann Intern Med 141:764-770 [PubMed] [Google Scholar]

6. https://www.invima.gov.co/images/nos_publican/2015/Marzo/Monitoreo_ Invima_2015-Mar-05.pdf. AUTORIDADES EN CALDAS ALERTAN POR VENTA DE MEDICAMENTOS SIN REGISTRO

7. $\mathbf{Z}$, Smith $\mathbf{P}$, et al. Combinations of potent topical steroids, mercury and hydroquinone are common in internationally manufactured skin-lightening products: a spectroscopic study. Clin Exp Dermatol 2016;41:196-201

8. Da Silva Balin, P. et al. Toxicological evaluation and anti-inflammatory potential of an ethanolic extract from Bromelia balansae (Bromeliaceae) fruit. Journal of ethnopharmacology, 2018; 79-86.

9. Kumar R, et al. Effect of Boswellia Serrata Extract on Acute Inflammatory Parameters and Tumor Necrosis Factor- $\alpha$ in Complete Freund ss Adjuvant-Induced Animal Model of Rheumatoid Arthritis. Int J Appl Basic Med Res. 2019; 9(2):100106.

10. Nieman LK. Recent Updates on the Diagnosis and Management of Cushing's Syndrome. Endocrinol Metab (Seoul). 2018 Jun;33(2):139-146. doi: 10.3803/ EnM.2018.33.2.139. PMID: 29947171; PMCID: PMC6021313

11. Reimondo G, Pia A, Bovio S, Allasino B , Daffara F, Angeli A, Terzolo M. Laboratory differentiation of Cushing's síndrome. Clinica Chimica Acta; International Journal of Clinical Chemistry. 2007, 388(1-2):5-14] DOI: 10.1016/j. cca.2007.10.036

12.UCSF. Clinical Labs-Chemistry, 1-12; tomado de: http://labmed.ucsf.edu/labmanual/db/resource/Centaur_Cortisol.pdf

13. Krasowski, M. D., et al. Cross-reactivity of steroid hormone immunoassays: clinical significance and two-dimensional molecular similarity prediction. BMC clinical pathology, 2014;14: 33. 